IV.

\title{
Bericht über die Verhandlangen der otologischen Section der British medical association in Glasgow am 8., 9. and 10. August 1888.
}

Von

\section{Dr. Thomas Barr.}

The British medical association visited Glasgow in August $8^{\text {th }}, 9^{\text {th }}, 10^{\text {th }}$ of this year and its Otological section furnished the first opportunity in Scotland for a gathering of Otologists. Dr. Thomas Barr of Glasgow presided in the Otological section, and the attendance of Otologists was unusually large. The subject of the presidential address was "the present position of the British School of Aural Surgery and the influence it has exerted upon the progress of the art, adding a few suggestions as to how that influence might be still further increased". (See copy of address sent.)

Three subjeets were selected for formal discussion, namely 1. The conditions calling for perforation of the mastoid portion of the temporal bone, and the best methods of operating. 2. Adenoid growths in the naso-pharynx; their influence on the middle ear, and their treatment. 3. The true value of these aids to hearing usually termed "Artifieial tympanic membranes".

Dr. P. Me Bride opened the discussion on operations on the Mastoid Process. He first discussed the therapeutic valne of incision of the soft-parts down to the bone in Mastoid inflammation before pus has formed. He believed that it was not a life saving operation, but chiefly palliative of pain and as a means of exploring the condition of the cortex. Incidentally Dr. M c Bride referred to the possibly bad effects of insufflation of powders in suppuration of the middle ear with small perforation of the membrane. He could not divest himself of the idea that in such cases mastoid inflammation sometimes results from pus retention due to these powders. In regard to the opening of the mastoid spaces, he would consider persistent pain, even although there 
be no swelling or redness over the surface, to be the main indication along with symptoms of meningeal irritation, with existing middle ear suppuration, and that the indication is commonly urgent just in proportion to the severity of the pain. In one case after chiselling through bone of almost ivory consistence for nearly one inch Dr. Mc Bride found, from the flow of dark venous blood, that the lateral sinus was wounded, but the haemorrbage was easily cheeked by an application of lint dipped in carbolic acid. This case did well even without any drainage from or opening into the mastoid antrum. The operation should be performed as close to the meatus as possible, and by means of a hammer and gouge. Trephines and borers are hardly justifiable. Much dressing is avoided and the wound is syringed with warm boracic lotion every bour or two, the fluid being made to pass both from the meatus to the wound and vice versâ. Dr. Me Bride suggested the use of the digestive ferments as trypsin and papayotin as a means of eleansing the recesses of the middle ear.

Dr. Stewart (Nottingham) said he should lay it down in a general way that, with local indications of pent up secretions in the middle ear causing injury to the general health, and not yielding to other metbods of treatment carefully carried out, the surgeon should penetrate the mastoid and establish a communication through the antrum mastoideum and the external meatus. The operations short of reaching the antrum, though, in themselves frequently of great benefit; were of secondary importance. He bad laid down rules for his own guidance for penetrating to the antrum mastoideum, and he hoped they would be found safe and reliable. If a line were drawn in the direction of the zygoma, and exactly coinciding with the osseus roof of the external meatus, and another downwards from that line at right angles to it, and exactly coinciding with the posterior osseous wall of the meatus, the posterior angle formed by these two lines would indicate the spot to be perforated. The perforation should be done as close up to these two lines as possible, but not beyond them. Let him compare these directions with other methods. The bony points being thus fixed upon, the incisions in the integuments should be guided accordingly. Bearing in mind the structures likely to be injured - lateral sinus, dura mater in the floor of the cranial fossa, facial nerve, and external semicircular canal - be believed it possible for the surgeon who proceeded with due eare to expose the dura mater without injuring it 
or without bad consequences to the patient. Beyond a fixed depth (five eighths of an inch) the surgeon should not go, and it was well known that in many skulls it would not be possible to bring the operation to a successful termination. The instruments that appeared to him to be the best were a trephine in preference to the chisel and mallet; sponges of two or three sizes, and a scoop. Dr. S tewart concluded by referring to some cases that had come under his own eare.

Dr. Barr referred to the propriety of opening into the antrum for the cure of those cases of chronic suppurative inflammation of the middle ear which had resisted all other methods of treatment, where, in spite of long continued and thorough cleansing and antiseptic treatment from the direction of the external anditory canal and Eustachian tube, the purulent discharge still continued and still emitted the odour of decomposition. By this means they might efficiently eleanse the cavities of the middle ear, and bring about an end to the secreting and decomposing process, and thus the removal of a serious menace to life itself. Dr. Barr believed that in this direction important work might be accomplished in the future. The desideratum was that an absolutely safe method of operation should be adopted. Dr. Barr did not think that operation by the mallet and chisel would insure the safety of the lateral sinus, if that sinus had an abnormally outer and anterior position. The blows of the mallet were very considerable especially in cases where the symptoms pointed to the possibility of cerebral abscess or meningitis, while to use chisel and mallet at the bottom of a deep wound was often very difficult. A proper dental burr, propelled by a suitable mechanism, was probably the safest instrument; was much easier than the chisel and mallet; and had not the dangerous points of the ordinary drill or trephine.

Dr. Laurence Turnbull (Philadelphia) was of opinion that they were apt to neglect the importance of constitutional treatment in mastoid disease such as diet, rest, use of antipyrin, aconite, iron, and iodine, and above all, the relief of pain by morphine and atrophine. Polypoid growths in a large majority of old cases should be removed, for dead bone was so often found under them. In illustration of this subject, Dr. Turnbull reported cases in which he had removed sequestra of bone from the mastoid and labyrinth, which had been covered up by the polypoid growths. He also gave an account of a case of tem- 
porary insanity following mastoid disease, in a man, where there was a hard, almost ivory like condition of the bone, which he removed but did not enter the antrum, and by this operation and constitutional treatment the case recovered.

Dr. Lew is (Birmingham) said cerebral abscess following suppuration of the middle ear was more frequently met with than was generally supposed. Five cases of death from this cause had come under his notice in five years; in two cases a postmortem examination was made, when in one a large quantity of pus was found in the temporo-sphenoidal fossa; the other case died from phlebitis of the lateral sinus. Two declined treatment, but the third, who suffered from old mastoid trouble, died, he was told, from symptoms of cerebral disease. Pain was not always to be the guiding condition that demanded either trephining the mastoid or skull, for a case came under his notice where most violent pain lasted for a considerable time. All operative treatment was deferred, and the ice-bag being applied for weeks, the patient made an apparent recovery. When, after cutting down upon the mastoid caries, was found, he thought, the mastoid should then be drilled; and, in one case, not having a drill at hand, he had used a gimlet with satisfactory result.

Dr. Walt on Browne (Belfast) referred to cases of caries of the mastoid process, and advocated free drainage by suction and passing a drainage tube through the mastoid process to the external meatus. He detailed two cases of abscess of the brain treated by trephining; one of these recovered. Dr. Browne did not approve of a drainage-tube in case of abscess of brain, and thought trouble was produced by it and washing out the abscess cavity. He would advocate simply trephining, opening the abscess and allowing it to drain away.

Dr. Jarqubar Mathes on spoke of the frequency of mastoid inflammation, and concurred in the classification of dividing this condition into two classes, namely, periostitis of the mastoid and ostitis of the whole bone. The first class was by far the most important, and occurred as a rule in children and young persons. This condition readily yielded to treatment. In the first outset leeches and fomentation were all that was required. This failing, incision through all the structures down to the bone must be made, followed by drainage. In ostitis, occurring oftenest in adult and old age, the trephine must be used to open the mastoid cells. 
Mr. James Black expressed pleasure at two points brought out by Dr. Me Bride, namely: 1. the risk of retention of inflammatory products due to blocking of the auditory passage by caking of a pack of boracic acid in the "dry method" of treating an otorrhoea, and the caution of vigilant attention of the aurist when this treatment was a dopted; and 2. the importance of pain and facial paralysis with recurrence of granulation polypus after removal as an urgent reason why perforation of the mastoid should be performed. Mr. Black showed a skull in which it would have been quite impossible to have performed the operation on the plan often recommended namely, a perforation of the bone half an inch behind and the same distance above the centre of the meatus without penetrating the wall of the lateral sinus. The perforator or drill was advocated, as dispensing with the jarring effect by which the use of the mallet and gouge was of necessity accompanied; but the only sure way of avoiding the lateral sinus was to hug the upper and posterior wall of the external anditory meatus.

Dr. Charles Warden said that with regard to the use of dry powders, boracic acid ete., and packing therewith, the difficulty was that one was not able to see the patients often enough, in which case the powder was left from day to day, not acting as an absorbent, but as a direct plug, which he considered most dangerous, as it simply locked a discharge of matter which should have a free exit. His preference was for boracic acid solution, zinc and carbolic etc. Dr. Me Bride, in reply, said that in spite of what had been said he must adhere to his riews as to the advantages of the ehisel. The objection as to doing harm by concussion were purely theoretical. The difficulties of diagnosing intracranial lesions, both as to their commencement and as to their nature were touched upon. As to constitutional treatment, he failed to see how it could do more than palliate; it could not produce drainage. Dr. Me Bride conld not agree with the President's suggestion as to mastoid opening in cases of perforation of the membrana-flaccida, as in such cases the suppurating cavity was often shut off from the tympanum, and could not be reached by through irrigation.

2. Adenoid growths in the Naso-Pharynx - their influence on the middle ear and their treatment.

Dr. Lennox Browne opened the discussion. He referred to the relation of adenoid growths to enlarged tonsils. Those 
growths often existed when the tonsils were not enlarged but they also frequently existed with enlarged tonsils, and the removal of the latter failed to remove the deafness because of the presence of the adenoid vegetations in the vault of the pharynx. It is important that these growths should be looked for in suppurative diseases of the ear, as well as non-suppurative cases. Dr. Browne advocated strongly the use of the finger-nail as the most efficient method of removing them. In regard to Anaesthetics, if the operation of removing the growths with the finger-nail be adopted they may be thoroughly cleared out of the nasopharynx by an introduction of the finger. If a second operation is required, which should be rare, it will usually be necessary in the case of a child to administer chloroform. Dr. Browne thought it probable the hypertrophic middle ear catarrh of the adult may sometimes be based upon the thickening of the submucous tissue of the nasopharynx induced by the adenoid growths of childhood and a limited experience warranted further trial of the process of scraping the post nasal space when there was thickening without actual out-growth, as an aid to the resolution of an adult hypertrophic middle ear catarrb.

The following papers connected with this discussion were also read. Dr. Hill on "The Roole of the Pharyngeal tonsil in health and disease", in which he advanced the view that its function is analogous to that of an ordinary lymphatic gland, namely the production of lencocytes for the blood, hence pathological conditions in this structure are associated with strumous. states of the system. Dr. A dolph Bronner of Bradford read apaper in which he stated that he found 8 per cent of 250 school children suffering from symptoms of adenoid growths. Of 152 cases of adenoid growths; 85 per cent showed symptoms of past or present affections of the middle ear. He believed the best method of removal was by Hartmann's Curette.

Dr. Jarquhar Matheson also read a paper on the relation of naso-pharyngeal diseases to stammering and stuttering. Several cases were related in which the removal of adenoid growths from the naso-pharynx completely cured the stammering and stuttering and Dr. Matheson believed that one of the following conditions was a constant factor in cases of stammering - namely, adenoid vegetations in naso-pharynx or enlargement of the turbinated bones, or chronic rhinitis.

In the discussion which followed these preliminary papers, 
Dr. Me Kenzie Johnston said there could be uo doubt that, if treatment of these growths were neglected, it would be impossible to cure either cases of middle ear catarrh or of suppurative disease. The middle ear is drained by the Eustachian canal; and therefore, if these growths prevented, the proper draining of the cavity, they failed to get one of the most important conditions - dryness and cleanliness - for the treatment of diseases of the ear. The effects of these growths on the general health were sufficiently serious to require careful consideration, but these hardly come under the subject of discussion. In conclusion, be expressed a decided preference for the forceps in place of the finger-nail so much lauded by Mr. Lennox Browne.

Dr. Howard said that while quite alive to the dangers in using anaestheties in operation on the naso-pharyx, he always used chloroform, and thought that with precautions against the flow of blood into the larynx, the danger could be reduced to a minimum. He specially emphasised the importance of drawing forward the head in introducing the finger, so as to relax the soft palate and give the maximum of space, and secondly throwing the head back and downwards over the edge of the table when the patient was under chloroform and the blood beg an to flow, the position of the head making it impossible for the blood to get into the larynx.

Dr. Laurence Turn bull (Philadelphia) was glad to have the opinion of Dr. Howard in reference to the danger of the use of chloroform in so comparatively trifling an operation, and the discussion bringing forth the information of the death of two individuals from this cause. He was of the opinion, and had so expressed it in his "Manual of Anacsthetics" that in such a class of operations systematic anaesthetics were not necessary, and sbould not be employed when they bad such an admirable local anaesthetic as the hydrochlorate of cocaine; he had found that all operations on the nose and throat could be successfully performed with a 2 or 4 per cent solution of cocaine; the cocaine was best dissolved in a 1 per cent watery solution of pure crystallised carbolic acid, which assisted the action of cocaine and prevented nervous symptoms.

Dr. Charles Denis on (Denver, Colorado) answered an enquiry, made by Mr. Lennox Browne, as to the frequency of these post nasal adenoid growths in his section of country. In so far as his experience went, he could not say that-either these 
growths or enlargements of glands generally were so frequently met with in the clear, dry atmosphere of Colorado as in sections bordering on the ocean.

Dr. Waxham (Chicago) simply agreed with Dr. Turnbull that adenoid growths were comparatively rare in America. His experience had been that the obstruction in the postnasal space which was frequently met with was due to bypertroply of the mucous membrane and of the pharyngeal tonsil ratber than to adenoid growths.

Mr. J a mes B lack believed the natural finger-nail, when well developed, was the best instrument, because it was in close contiguity to a tactile organ; but still, the fact of some men, that is, Dr. Bronner, disearding the finger-nail in favour of the curette seemed to show that in certain cases some other instrument was necessary. In most cases, however the natural or artificial nail was necessary to complete the operation after an other instrument has been used for the removal of the adenoid growths in the naso-pharynx, and the reason why was, that the choanæ were often blocked by thickening of the mucous membrane around these openings, and it was his (Mr. Black's opinion) that the only safe way to remove this thickening was by the use of the nail.

Dr. Jarqubar Mathes on said that tho removal with the finger-nail was preferable to the use of any instrument. An anaesthetic ought to be carefully administered and a weak alkaline solution for nasal injection to be continued for some weeks after.

Dr. Walter Wolston (Edinburgh) said that a very large percentage of the deafness now existing in Great Britain, his experience would venture to say above 75 per cent, was due to the presence of adenoid vegetations in the naso-pharynx. He used Loewen berg's forceps to clear the larger masses, and then, with the finger-nail, completely seraped out the choane, vault, and pharynx, and various crypts and recesses of the post-nasal space, paying particular attention to the little eushion that almost always lay just above and pressed on the extremities of the Eustachian tubes. The finger-nail alone would not suffice in all cases, as in those above seven years of age the growths, often of a fibrous character, which sprang from the posterior wall of the pharynx, were so embedded in the normal tissues that a considerable amount of a cutting wrench with the forceps was ne- 
cessary. It was well to reduce the posterior turbinated bodies if they were very large, and this he did by the galvano-cautery loop passed through the anterior nares.

Dr. Barr (the President) thought the men who brought this subject before the profession, such as M e y er, Guye and Loewenberg had performed a most important service to aural surgery; indeed the discovery of these growths as sources of ear disease marked an important epoch in the development of the knowledge of ear disease. There was no doubt that these growths were very frequently met with in Scotland, and were most important causes of ear disease.

In their operative treatment Dr. Barr employed finger-nail, artificial stcel nail, and Loewenberg's forceps, or modifications of them. He preferred as a rule, the steel nail and the forceps to the finger-nail. The latter was not sufficient to remove the tough growths, and was not so cleanly a method as instruments. Dr. Barr frequently employed first the forceps and then finished with the artificial nail. The introduction of proper forceps into the naso-pharynx was less painful than the finger, but the sensitivenens varied very much in different persons. Local application of cocaine was very useful, but it was well to avoid chloroform, although in very nerrous children this anaesthetic was necessary. If chloroform was employed, the head should he placed well down so as to avert the possibility of blood entering the windpipe. One operation was not usually sufficient to completely clear out the vegetations, and three or four might be necessary before a smooth condition of the mucous membrane and a permeable state of the nasal channels were secured.

Mr. Lennox Browne, in reply, referring first to the question of anaesthesia, repeated that if operation were done at the time of first examination, no anaesthetic was required. Although the effect of introducing the finger into the naso-pharyngeal vault was disagreeable, and, in young children, somewhat terrifying, it was by no means really painful, and local anaesthesia by means of a cocaine spray of 10 per cent strength was quite sufficient to annul all sensation. Although be preferred the finger nail for removal of these growths, and had never found a case in which it could not be employed with success, be desired to accord the greatest liberty of action to every one onthis point. The important thing was not what instrument was used, but how to attain 
a good result; and, personally, he very rarely found it necessary to repeat an operation by this method, while three or four repetitions of procedure by forceps had been admitted by some speakers.

3. The true value of those aids to hearing usually termed "Artificial tympanic membranes."

Dr. W. Laidlaw Purves introdnced the discussion. He said that the artificial drum head acts as a protector, as a vehicle for medicaments, as an absorbent; as a moistener; but it is preeminently as a tension transposer, and an assistant to the intra-tympanic muscles that it effects the changes which delight both patient and practitioner. The artificial membrane does for the ear what the artificial lens does for the eye, performing the work which the muscles themselves cannot do witbout a strain, so that those wonderfully minute variations necessary for conversational hearing may be effected with comfort.

In connection with this discussion Dr. Lanrence Turnbull of Philadelphia next read a paper in which the following conclusions were expressed. 1. That such aids were important to the bealth of the ear, by preventing dryness and the general danger to the hearing, from the want of the protecting power of the natural membrane. 2. A certain degree of hearing would be possible without the membrana tympani, but perfect hearing was impossible without it. 3 . In the rarious ageneies which had been employed they had not only the means of protecting and preventing drying effects of the air, but also the prevention of the passage into the middle ear of injurions foreign agents, the prevention of disease from cold air or water, so apt to set up acute inflammation, followed by abscess in the mastoid or brain. Satisfactory results bad been obtained from the cotton ball or pellets of "Yearsley". The objection to this was the tendency which the ordinary cotton had to cause irritation, by bearing in its fibres bacteria and micrococei; also other foreign matters. Again it sometimes fitted so closely, owing to discharge or mucus on its surface, as to make a shut sac, and absolutely prevent the vibrations of the membrane, thus acting as a damper. These difficulties were overcome by employing corrosive sublimate soltutions with "sublimate cotton", or a disc of sublimate gauze, moistened with fluid cosmoline, so as to make it more adhesive. When water with glycerine was employed the mixture 
would soon ferment in the car and become irritating and cause inflammation. By treatment they could much sooner employ the artificial membrane, even when there was a slight suppuration going on. The solution of the sublimate should not exceed in strength 1 to 4,000 ; if stronger it gave pain. The fluid cosmoline, or vaseline, was to moisten the pellet when about to be introduced, if the parts were dry. The pellets were placed in position by means of ordinary tweezers; the thread must be cut off close, so as not to be seen. He had discarded all the forms of apparatus which had any metallic spring, handle, etc., having found them always irritating and injurious.

As bearing on this subject Dr. Ellis (Newcastle) read a paper on the "Fraudulent treatment of the Deaf by Impostors". Dr. Ellis showed artificial drums, brought to him by a working man, and for which he paid $₹ 2-17-6$, which he stated be could very badly afford at the time, but which he was induced to send by the positive promises of cure beld forth. $\mathrm{He}$ (Mr. Ellis) had the metallic portion of these drums examined by a goldsmith, and he pronouneed them "brass", which he thought they would agree with him was a most appropriate metal. The poor man who was induced to purchase these drums suffered from nervous deafness and tinnitus, and had nothing whatever the matter with his tympanum or conducting apparatus, and Mr. Ellis purposely avoided entering into the question of the applicability of the artificial tympanum, but from the various impositions practised under its cover, be was sometimes inclined to ask, was the artificial tympanum a blessing or a curse? Might they not, failing to cure some of these patients, at least endeavour to save them from imposition and fraud by exposing the nefarious schemers who lay in wait for them?

Dr. Mathes on said that the most useful form of artificial membrane was the cotton wool-plug (antiseptic) and a covering or plug of boric acid. All dises of tissue of whatever variety acted as irritants, and should be used with much caution.

Dr. Donald Stewart said the difficulty of deciding whether the artificial membrane was a means of avoiding dampness of the membrane or a means of introducing the necessary moistness was an important point. In bealth the membrane was quite dry, and they had thus a pregnant hint to take care not to overtreat these cases. Dr. Warden said his preference was for Field's drums or a modification of Toynbee's or Yearsley's. 
Mr. Richard Ellis had not found the artificial tympanum of much use in hospital practice, but in a number of cases in private practice it was undoubtedly serviceable.

Dr. Walter Downic was in the babit, after all suppuration had ceased, of using a layer of cotton-wool firmly compressed between finger and thumb, and applied by a pair of fine forceps; these could afterwards be readily applied by the patient himself. Should this not succeed, he frequently used Fiel d's pattern. Lately be had partly as an experiment, been applying a small circular patch of pellicle of egg, which was soft and lay firmly in contact with the membrane.

Dr. James Erskine had found Professor Lucae's artificial tympanic membrane very useful. It had the advantage of being very easily inserted, and no injury could happen to the meatus in its introduction. He bad come to the conclusion that the cotton wool pellets of Yearsley's invention was the best artificial drum membrane.

Dr. J. Maefic said that we need not expect to find one form of artificial drumbead applicable to all cases amenable to improvement by this treatment, the form varying with the case, but he thought the most generally applicable was Yearsley's cotton-plug, or some modification of it.

Dr. Barr said the cotton pellet was on the whole the best form of artificial tympanic membrane. The india rubber dise was, however, occasionally, though rarely, more efficient. As usually sold by the instrument maker it was very defective, as from the mode of connection of the dise with the stem a hard metal knob pressed upon the tympanic structures. Before employing this aid to hearing, the purulent secreting process, if present, should be cured, or should be reduced to a very small compass, and great care should be taken at first that it should remain in the ear only for a short time, not more than two or three hours, otherwise a fresh purulent process might be excited which would greatly discourage the patient. It should rarely remain in the ear during the sleeping hours. Before deciding as to the value of this remedy in any given case, we must take great pains to try more than once, various sizes and shapes, in various positions. He believed that many failures were accounted for by the want of sufficiently painstaking efforts in those directions. A certain degree of pressure was usually necessary before the good effect was achieved, and while the postero-superior quadrant was usu- 
ally the best position, trial should be made over the short process of the malleus, and indeed on any part in the upper regions of the tympanum. The pellet should be moistened, preferably by some thickish antiseptic fluid. In some cases where the cotton pellet or dise was apt to become misplaced, falling out from its proper position, it was a good thing to immerse the cotton in collodion and apply it to the part. In this case, if the ear could bear prolonged contact, it need not be removed for days or weeks. He knew a patient who kept it in the ear day and night for months when the collodion was applied. Dr. Barr expressed the opinion that the so-called "ear-drums", so extensively advertised in the newspapers, and which consisted of a piece of India-rubber attached to a metallic stem, were frequently highly injurious to persons with defective hearing, that the number of deaf persons who might be benefited by this form was comparatively small, and that in no case should an artificial drum of any form be used, until a surgeon had examined the defective ear and ascertained if the case was suitable, for if not suitable their introduction into the ear was not merely valueless but decidedly injurious.

The view was expressed by several members of the section that this expression of opinion by the President regarding the "ear drums" as commonly advertised, should be sent to the Glasgow newspapers for insertion. The secretaries were requested to carry this out.

In addition to these formal subjects of discussion the following other communications were made to the section.

Cerebral abscess from ear disease. Dr. Barr showed two cases which had been operated upon by Dr. Macewen for cerebral abscess due to ear disease. The first has been already described in the Archives of Otology (Volume XVI. p. 146).

The second case was that of a young man, aged 21, who was operated uponon the $13^{\text {th }}$ of May last. There had been a discharge from the left ear for eight or nine years. For three weeks before the operation the symptoms were headache, vomiting, slow and intermittent pulse, normal or subnormal temperature, contraction of left pupil, paresis of all the ocular muscles with exception of the external rectus, partial facial paralysis on the opposite side, paresis of right arm with wrist-drop. The mastoid was first trephined, so as to establish communication with the external anditory canal, but little or nopus was found 
there. Dr. Macewen then trephined above the external anditory canal, and pierced the brain-tissue with a trocar. Three ounces of horribly foetid pus were removed. The eavity was syringed with a weak solution of carbolic acid. A chicken bone drainage tube was inserted. The dressing was not removed for three weeks, when the wound was found to be almost healed. The patient recovered without a single bad symptom. The opening was filled up with firm bone, and the patient was now perfectly well.

Dr. Charles Warden (Birmingham) related a case of congenital ear disease, producing mastoid abscess and facial paralysis. This was a child in whom a discharge from left ear was noticed shortly after birth, followed in a short time by convulsions producing paralysis of the left side of face; soon after a large abscess formed at the back of the ear in the temporal and mastoid region; when opened diseased bone was evident. Several pieces of dead bone were subsequently removed from the meatus. Ultimately the facial paralysis completely passed off. Dr. Warden was of opinion that a low form of inflammation had been going on from birth.

Dr. Christopher Lewis (Birmingham) recorded a case of deep cellulitis of the neck with partial paralysis of right arm following acute otitis media caused by a blow. A boy, 14 years of age, fourteen days after a blow on the ear, was seized with pain in ear and head, vomiting, delirium and high temperature. About the ninth or tenth day swelling and redness appeared over the apex of mastoid followed by deep seated inflammation of neck. The membrane had a boggy appearance but there was no perforation, but marked deafness. An enormous swelling as hard as a board developed in the neck. There were no rigors, but there was intense pain. Some difficulty of movement was noticed in the right arm owing to the injury received by the cervical plexus. An incision was made a few inches above the clavicle and a grooved probeintroduced which first came upon a caseous mass and then there was a discharge of pus. From this time the symptoms steadily subsided. The recovery has been complete and the bearing has become normal. The probability is that suppuration in the mastoid cells first took place, the pus breaking through the inner surface of the apex and thus underneath the sterno-mastoid muscle into the deep parts of the neck. 
Bericht über die Verhandlungen der otologischen Section in Glasgow. 55

Dr. J. Erskins showed 1, sequestra of portions of labyrinth from a woman who suffered from a discharge from right ear for 4 years, there were facial paralysis of the same side and total deafness. The sequestra included portions of cochlea, portion of inner wall of tympanum and ineus. 2. An unusually large auricular appendage. This was a projection in front of the right ear of a woman aged 29. The base of the projection measured half an inch and its length over an inch. Under chloroform it was removed by one suip of the scissors. 